\title{
Comparison of VLES and LES Turbulence Modeling for Swirling Turbulent Flow
}

\author{
P. Tiwari ${ }^{1}$, Z. Xia ${ }^{1}$ and X. $\operatorname{Han}^{1,2 \dagger}$ \\ ${ }^{1}$ College of Energy and Power Engineering, Nanjing University of Aeronautics and Astronautics, Nanjing \\ 210016, China \\ ${ }^{2}$ Aero-engine Thermal Environment and Structure Key Laboratory of Ministry of Industry and Information \\ Technology, China
}

†Corresponding Author Email: xshan@nuaa.edu.cn

(Received August 9, 2019; accepted December 30, 2019)

\begin{abstract}
Swirling flow has been widely used in gas turbine and aero-engine combustor to stabilize the flame. However, accurate numerical prediction of the swirling turbulent flow is difficult due to complex vortex movement in the flow, and turbulence modeling is a key factor. To assess the turbulence modeling in predicting the swirling flow, numerical studies are conducted for a well-documented swirling flow case. Three turbulence models are applied in the framework of scale resolved models, i.e. a newly developed VLES (Very-large eddy simulation) model, two LES (Large eddy simulation) models including the WALE (Walladapting local eddy viscosity model) and CSM (Coherent Structure Method). Numerical results are compared with the experimental results including the mean and RMS velocities. It is found that VLES model performs best among the three models and the other two LES models give comparable predictions. The complex vortex structures are explored based on the unsteady simulation results. The study demonstrates the high potential of VLES modeling for accurate prediction of complex swirling flow.
\end{abstract}

Keywords: Very large eddy Simulation; Swirling flow; Unsteady flow; Turbulence model.

\section{NOMENCLATURE}

$k \quad$ turbulent kinetic energy

$L_{c} \quad$ turbulent cutoff length scale

$L_{i} \quad$ integral length scale

$L_{k} \quad$ Kolmogorov length scale

$p$ pressure

Re Reynolds number

$S \quad$ swirl number

$U$ the axial velocity

$W \quad$ the tangential velocity

\section{INTRODUCTION}

Clean and complete combustion has emerged as key aspect for researchers to investigate over long time. In recent years, modern gas turbine and aero-engine combustor request higher standard for emissions (Gupta et al. 1984; Shah et al. 2019). Effective combustion design is a key factor. In those situations, swirling flow is widely applied, which is applied to stabilize the flame and also for a better mixing of the fuel and air.

Swirling flow has been developed decades ago, $\varepsilon$ the dissipation rate of turbulent kinetic energy

$\mu \quad$ molecular viscosity

$\mu_{t} \quad$ turbulent viscosity

$\rho$ density

$\sigma_{k,} \sigma_{\omega} \quad$ model constant

$\omega$ specific dissipation such as in the study by Krain (1988), where experimental measurements showed the distinct swirling flow character in the blade passages of the rotor. The strength of the swirl is generally described by the swirl number $S$, defined as the ratio of the angular momentum to the axial momentum (Gupta et al. 1984). It is found that, when the swirl number is larger than 0.6, there exists vortex break-down in the swirling flow. Vortex movements have been extensively studied by experimental, numerical and theoretical methods (Gupta et al. 1984; Shtern and Hussain, 1999; 
Wang et al. 2019). A complex and fundamental phenomenon found in the swirling flow is the unsteady and periodic motion of large-scale coherent structures. The presence of turbulence at high Reynolds number further complicates the flow mechanisms. Understanding and characterizing turbulent swirling flow is one of the fundamental challenges in fluid mechanics.

The explosive growth in the computer ability gives an opportunity for the researchers to investigate the complex phenomena of swirling turbulent flow using numerical methods. As to the turbulence modeling in the field of Computational Fluid Dynamics (CFD), the methods can be roughly divided into three major categories, i.e. ReynoldsAveraged Navier-Stokes (RANS), Large Eddy Simulation (LES), and DNS (Direct Numerical Simulation), according to the decreasing level of turbulence modeling. For swirling flow, due to the highly unsteady, complex turbulence scale evolution flow nature, the predictions of URANS methods generally have poor accuracy (Gyllenram, et al. 2007). LES has attracted extensive interests for various complex turbulent flow simulations as it can accurately resolve the large scale coherent turbulent scales. In terms of DNS, to accurately capture the turbulence scales for such swirling flow, it requires very fine mesh resolution. The mesh with 2.6 billion unstructured cells has been applied for a DNS study of swirling flow at Reynolds number of 40000 (Moureau et al. 2010). Thus it is still too computationally expensive to use DNS to study the swirling flow at the Reynolds number in the order of $10^{4}$.

In recent years, new self-adaptive turbulence models, such as VLES (Very-Large Eddy Simulation) (Han and Krajnovic, 2013; Han and Krajnovic, 2015) and PANS (Partially-Averaged Navier-Stokes) (Girimaji, 2006; Saroha et al. 2018), are of growing interests in the hybrid RANS-LES modeling for applications to the complex turbulent flow of industrial relevance. The VLES method is a unified simulation approach enabling a seamless evolution from RANS to LES and finally approaching DNS depending on the numerical resolution. The VLES method works quite efficiently for several canonical high Re turbulent flows (Han and Krajnovic, 2013; Han and Krajnovic, 2015).

Even though many numerical studies have been carried out for swirling flow, there are a number of aspects which need to be discovered. The main purpose of the present study is to evaluate the capability of VLES and traditional LES in predicting the complex swirling flow with comparisons of available experimental data. The experiment selected in the present study is a classical swirling flow case conducted at the University of Cambridge (Sweeney, et al. 2012).

The paper is organized as follows. The numerical details of the VLES and LES calculations are firstly presented. Following is the computational results obtained from the present VLES and LES models as well as the comparisons with available experimental data. Finally, major findings of the current work are summarized in the conclusion part.

\section{NumericAl MethodS AND SETUPS}

The numerical methods and setups for the present VLES and LES calculations are described in this section.

\subsection{Governing Equations}

In the framework of the present VLES and LES calculation, the filtered Navier-Stokes equations can be expressed as:

$\frac{\partial \rho}{\partial t}+\frac{\partial \rho \bar{u}_{i}}{\partial x_{i}}=0$

$\frac{\partial \rho \bar{u}_{i}}{\partial t}+\frac{\partial \rho \bar{u}_{i} \bar{u}_{j}}{\partial x_{j}}=-\frac{\partial p}{\partial x_{i}}+\frac{\partial}{\partial x_{j}}\left[\mu\left(\frac{\partial \bar{u}_{i}}{\partial x_{j}}+\frac{\partial \bar{u}_{j}}{\partial x_{i}}\right)-\frac{2}{3} \delta_{i j} \frac{\partial \bar{u}_{l}}{\partial x_{l}}\right]$

$+\frac{\partial}{\partial x_{j}}\left(-\rho \overline{u_{i}^{\prime} u_{j}^{\prime}}\right)$

The subgrid scale stresses in Eq. (2) are unclosed terms to be modeled. The modeling methods are given below as:

$-\rho \overline{u_{i}^{\prime} u_{j}^{\prime}}=\mu_{t}\left(\frac{\partial \bar{u}_{i}}{\partial x_{j}}+\frac{\partial \bar{u}_{j}}{\partial x_{i}}\right)-\frac{2}{3}\left(\rho k+\mu_{t} \frac{\partial \bar{u}_{k}}{\partial x_{k}}\right) \delta_{i j}$

Based on the modeling, the unknown parameter involved in the above equations is only the turbulent eddy viscosity (i.e. $\mu_{t}$ ). Note that it is the subgrid scale eddy viscosity in the present study.

\subsection{Very-Large Eddy Simulation Modeling}

In the present study, the VLES method is applied for the swirling flow. It is a seamless modeling method which can change from RANS to DNS seamlessly depending on the resolution mesh scale. Between the two limits of RANS and DNS, it is named as VLES. The general development details can be found in previous references (Han and Krajnovic, 2013; Han and Krajnovic, 2015). Here the modeling is briefly described as follows.

The present VLES modeling is accomplished with the underlying RANS turbulence model of the BSL (Baseline) $k-\omega$ model, i.e. VLES- $k \omega$ model. The governing equations of $k$ and $\omega$ are exactly the same as in the BSL $k-\omega$ RANS model. They can be expressed as:

$$
\begin{aligned}
& \frac{\partial \rho k}{\partial t}+\frac{\partial \rho \bar{u}_{j} k}{\partial x_{j}}=P_{k}-\beta^{*} \rho k \omega+\frac{\partial}{\partial x_{j}}\left[\left(\mu+\sigma^{*} \mu_{t}\right) \frac{\partial k}{\partial x_{j}}\right] \\
& \frac{\partial \rho \omega}{\partial t}+\frac{\partial \rho \bar{u}_{j} \omega}{\partial x_{j}}=\alpha \frac{\omega}{k} P_{k}-\beta_{1} \rho \omega^{2}+\frac{\partial}{\partial x_{j}}\left[\left(\mu+\sigma \mu_{t}\right) \frac{\partial \omega}{\partial x_{j}}\right] \\
& +2 \rho\left(1-F_{1}\right) \sigma_{\omega 2} \frac{1}{\omega} \frac{\partial k}{\partial x_{j}} \frac{\partial \omega}{\partial x_{j}}
\end{aligned}
$$

The present VLES- $k \omega$ modeling only modifies the formulation of the eddy viscosity, i.e.:

$\mu_{t}=F r \cdot \rho \frac{k}{\omega}$

where $F r$ is the resolution function, which is one of 
the cores in VLES modelling. Here it is modelled as:

$F r=\min \left(1.0,\left[\frac{1.0-\exp \left(-\beta L_{c} / L_{k}\right)}{1.0-\exp \left(-\beta L_{i} / L_{k}\right)}\right]^{n}\right)$

where $\beta$ and $n$ are the model constants. It can be seen that three turbulence length scales are involved in Eq. (7), i.e. $L_{c}, L_{i}$, and $L_{k}$, which are the turbulent cut-off length scale, integral length scale, and Kolmogorov length scale, respectively, defined as:

$L_{c}=C x(\Delta x \Delta y \Delta z)^{1 / 3}$

$L_{i}=k^{3 / 2} /\left(\beta^{*} k \omega\right)$

$L_{k}=v^{3 / 4} /\left(\beta^{*} k \omega\right)^{1 / 4}$

All the model constants involved in Eqs. (4)-(8) are given in Table 1.

Table 1 Model constants for the VLES- $k \omega$ turbulence model

\begin{tabular}{|c|c|c|c|c|}
\hline \multirow{2}{*}{} & \multicolumn{4}{|c|}{ Parameters } \\
\cline { 2 - 5 } & $\beta$ & $n$ & $C x$ & $\beta^{*}$ \\
\hline value & 0.002 & 2.0 & 0.61 & 0.09 \\
\hline
\end{tabular}

\subsection{LES -WALE Model}

To further validate the VLES method, LES is also applied in the simulations. Here, the widely used WALE (Wall-Adapting Local Eddy-viscosity) model (Nicoud and Ducros, 1999) is applied. It has the following form:

$\mu_{t}=\rho \Delta_{s}^{2} \frac{\left(S_{i j}^{d} S_{i j}^{d}\right)^{3 / 2}}{\left(\bar{S}_{i j} \bar{S}_{i j}\right)^{5 / 2}+\left(S_{i j}^{d} S_{i j}^{d}\right)^{5 / 4}}$

where the model parameters are:

$\Delta_{s}=C w(\Delta x \Delta y \Delta z)^{1 / 3}$

$S_{i j}^{d}=\frac{1}{2}\left(\bar{g}_{i j}^{2}+\bar{g}_{j i}^{2}\right)-\frac{1}{3} \delta_{i j} \bar{g}_{k k}^{2}$

$\bar{g}_{i j}=\frac{\partial \bar{u}_{i}}{\partial x_{j}}$

The WALE model has been designed to produce the correct wall asymptotic variation of the subgrid scale viscosity and thus it needs no damping functions. Those make the WALE model suitable for the unsteady turbulent flows with complex turbulence structure.

\subsection{LES -CSM Model}

Another LES model applied in the present study is the Coherent Structure Model (CSM) developed by Kobayashi (2005). The CSM model is developed using a coherent structure function defined as the second invariant normalized by the magnitude of a velocity gradient tensor. The modeling can be written as:

$\mu_{t}=\rho C \Delta^{2}|S|$

where the model parameters are:

$$
\begin{aligned}
& \Delta=(\Delta x \Delta y \Delta z)^{1 / 3} \\
& C=C_{2}|F c s|^{3 / 2}(1-F c s) \\
& F c s=\frac{Q}{E}=\left(-\frac{1}{2} \frac{\partial \bar{u}_{i}}{\partial x_{j}} \frac{\partial \bar{u}_{j}}{\partial x_{i}}\right) /\left(\frac{1}{2} \frac{\partial \bar{u}_{j}}{\partial x_{i}} \frac{\partial \bar{u}_{j}}{\partial x_{i}}\right)
\end{aligned}
$$

The models satisfy a correct asymptotic behavior to a wall. It is applicable not only to turbulent flow but also to laminar flow. It is thus suitable for complex swirling turbulent flow. Recent researches (Bennasr, et al. 2017; Li, et al. 2017) have shown increasing interests in the model. It is thus selected in the simulation to validate the VLES modeling.

\subsection{Numerical Setup Details}

The present VLES model and the CSM model of LES are implemented in a general CFD code, ANSYS Fluent (ANSYS Fluent, 2015), based on finite-volume method, which is adopted for the present numerical simulations. The VLES turbulence modeling and the CSM model are implemented in the code via UDFs (User Defined Function). The convective terms are discretized using a central differencing scheme coupled with a small fraction of upwind scheme. The second-order upwind scheme is used for the turbulence model equations, and a second-order implicit scheme is used for the temporal discretization. The SemiImplicit Method for Pressure-Linked Equations (SIMPLE) algorithm is used for pressure-velocity coupling. The time step is $3.0 \times 10^{-5} \mathrm{~s}$, resulting in the CFL number smaller than 0.6 at most of the regions. The simulations are averaged for more than 15000 time steps after the initial transient development for about 15000 time steps.

\subsection{Boundary Conditions}

The computational domain in the present study includes $25 \mathrm{~mm}$ upstream of the bluff body in order to better capture the inflow conditions. The domain extends $300 \mathrm{~mm}$ downstream of the bluff body in the axial direction and $200 \mathrm{~mm}$ in the radial direction. Schematic of the swirling flow rig is shown in Fig. 1(a) with the coordinates and the present simulation is performed under the Cartesian coordinates. The hexahedral mesh used, a slice of which is shown in Fig. 1(b), consists of approximately 3.2 million cells after several numerical tests ranging from about 1.0 million to about 5.0 million cells. Based on the mean and rms velocity comparisons on different meshes, it is found that the results show no significant differences when the mesh contains more than 3.2 million cells. Thus the mesh with 3.2 million cells is finally selected. The computational grids are comparable to those in previous LES studies (Brauner, et al. 2016) of the same swirling case.

The boundaries consist of three inlets, one outlet and one free stream boundary. The flow in the outer annulus has a mean velocity of $U_{o}=18.7$ $\mathrm{m} / \mathrm{s}$. In the inner annulus, it has the velocity of $U_{i}$ $=8.7 \mathrm{~m} / \mathrm{s}$ and the co-flow air of $U_{c o}=0.4 \mathrm{~m} / \mathrm{s}$. The Reynolds numbers derived from the bulk velocities and the exit geometry are $\mathrm{Re}_{i}=5960$ for the inner annulus and $\mathrm{Re}_{\mathrm{o}}=11500$ for the outer 
annulus. There are several swirling cases experimentally studied in the measurements in the Cambridge University (Sweeney, et al. 2012; Barlow, et al. 2012). The present study chooses the highest swirling flow case, cSwB3, in which the swirling number is $S=0.79$ located in the outer annulus. Extra RANS simulations are performed in order to get the inflow velocity distributions which can well match the measurements at a very close position to the bluff body surface. Also, the turbulent kinetic energy, $k$, and the dissipation rate ratio, $\omega$, obtained from the extra RANS simulations are imposed for boundary conditions for turbulence equations at the inlet. The "Vortex Method" is also applied to generate the synthetic turbulent fluctuations at the inlet.

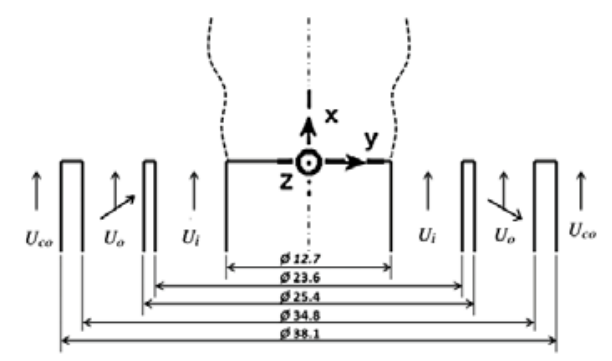

(a)

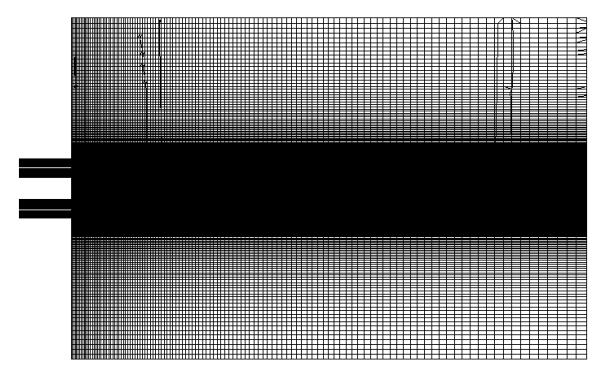

(b)

Fig. 1. Schematic of the swirling flow rig (a) and the computational mesh (b) in the central plane (a cut from the 3D geometry).

\section{RESUlts AND Discussions}

\subsection{Mean Velocity Results}

Figure 2 shows the calculated mean velocities at various locations from the inlet. The results are for three different turbulence models compared with the experimental results (Sweeney, et al. 2012; Barlow, et al. 2012). They are at four different distances from the inlet, i.e. $0.2 \mathrm{~mm}, 10 \mathrm{~mm}, 30$ $\mathrm{mm}$ and $50 \mathrm{~mm}$, respectively. Those regions can be roughly classified as near $(0.2 \mathrm{~mm} \& 10 \mathrm{~mm})$, intermediate $(30 \mathrm{~mm})$ and far region $(50 \mathrm{~mm})$. Through the comparisons of VLES predictions and the experiments, the predicted results of VLES agree well with the experimental data in the near region (i.e. the distances of $0.2 \mathrm{~mm}$ and $10 \mathrm{~mm}$ ), but as the distance increases, there exist some deviations from the experiments. The reasons attributed to this could be the swirling inflow velocity and turbulence effects. Recent studies (A1Abdeli \& Masri, 2015; Chen, et al. 2016) show that the swirling flow is sensitive to the swirling generator. The present study applies the mean velocity of the swirling flow, but the turbulence at the inlet is not accurately given due to unclear geometry of the swirling generator in the experiments. Another reason could be that swirling flow is challenging for turbulence modeling due to the complex turbulence vortex breakup and decay process. The results in Fig. 2 also show the numerical predictions by the LES-WALE and LESCSM models. The two LES models generally give reasonable predictions compared with the experiments. However, the more deviated results of LES can be seen compared with VLES, especially at the intermediate and far region. It implies that VLES gives a promising result with the experiments overall and LES models give reasonable results in the near region. In the far downstream region, the turbulence modeling has big effects on the numerical accuracy.

Another observation from Fig. 2 is that the radial mean velocity component (Vmean) exhibits a larger deviation between the numerical and experimental results. This is probably due to the inaccurate setup of swirling flow inlet, i.e. the accurate modeling of swirler is important for the accurate simulations. The swirler should be included in further simulations in order to improve the accuracy of the inlet boundary conditions. Further, the LES-WALE model produces a higher prediction at the near region indicating a slower decaying swirling flow predicted by this model, for the present simulation.

Figure 3 represents the contours of the mean velocities by different turbulence models. As can be seen from the figures, the mean velocity contours generally agree with each other by different turbulence models, although there are some differences locally. From the axial mean velocity results, it can be seen that the spreading of the jets from the outer annuals is slightly different by different models. The LES-WALE model (Fig. 3(a)) gives the largest spreading angle, the LESCSM model (Fig. 3(b)) gives the smallest, and the VLES model (Fig. 3(c)) in between. The results in Fig. 2 of the mean axial velocity at $30 \mathrm{~mm}$ and 50 $\mathrm{mm}$ also show that the VLES predictions are in between of the LES-WALE and LES-CSM model, and the VLES results are closer to the experimental data. Thus, it can be concluded the contours of VLES results are closer to the real physical flow field. For the mean tangential velocity, the contours of VLES are close to those of LES-CSM, while slightly different from LES-WALE in the far downstream region. The results in Fig. 2 of the mean tangential velocity also show that the VLES predicts the best of the tangential velocity, and LES-WALE the worst. Those results demonstrate that the VLES contours can be more trusted as the real physical flow field of the complex swirling flow, for the present test cases. 


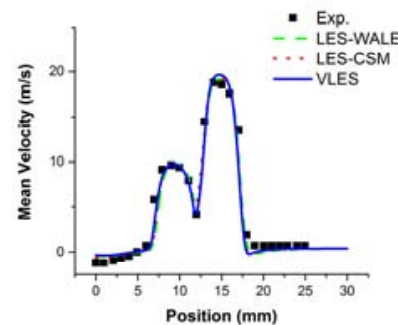

Umean@02 mm

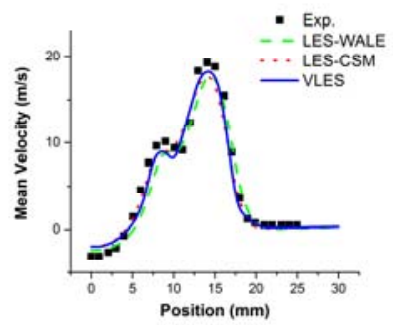

Umean@10 mm
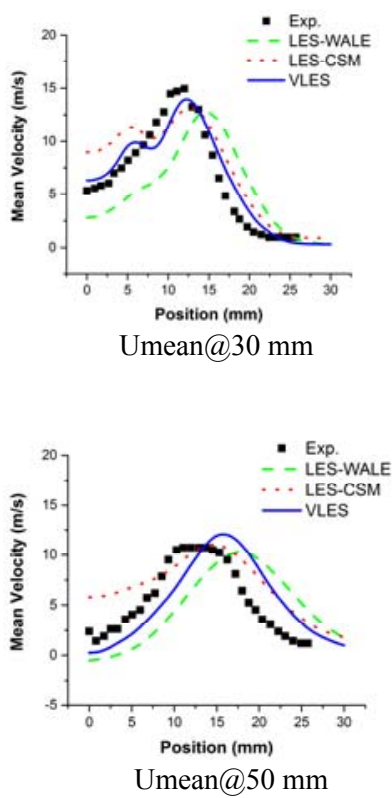

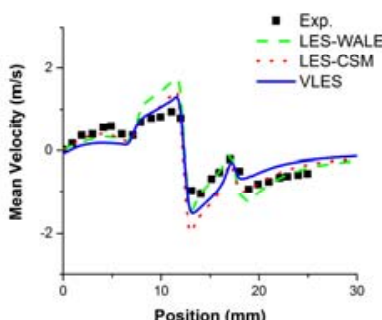

Vmean@02 mm

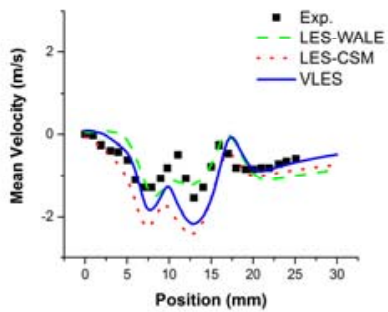

Vmean@10 mm

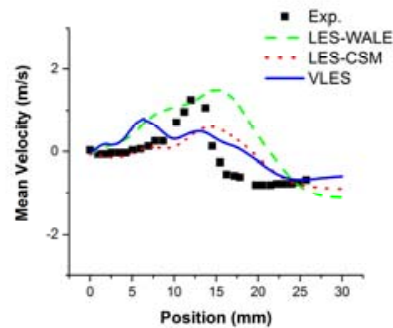

Vmean@30 mm

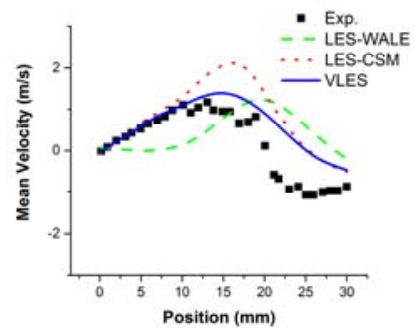

Vmean@50mm

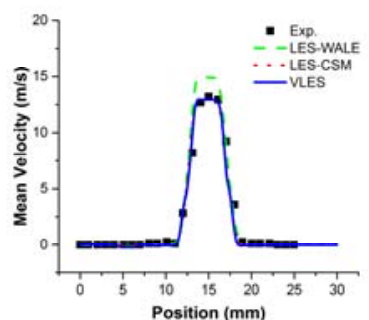

Wmean@02 mm

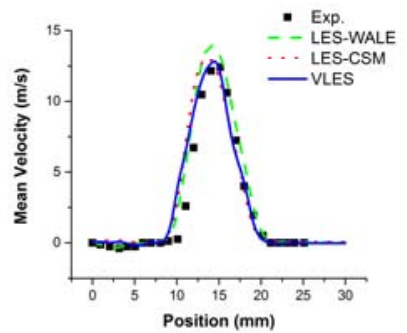

Wmean@10mm

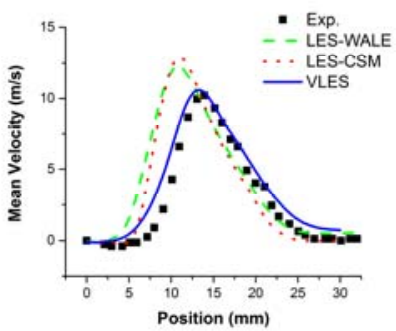

Wmean@30mm

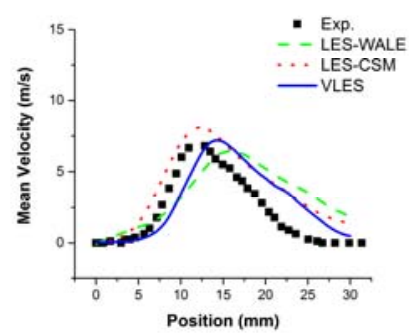

Wmean@50mm

Fig. 2. Comparison of axial (U), radial (V) and tangential (W) mean velocities for the swirling turbulent flow with different models.

\subsection{RMS Velocity Results}

Figure 4 shows the calculated rms (Root Mean Square) velocities along the axial, radial and tangential directions at various locations. The results are also divided for three regions near $(0.2$ $\mathrm{mm} \& 10 \mathrm{~mm})$, intermediate $(30 \mathrm{~mm})$ and far region $(50 \mathrm{~mm})$. The first observation is that the distribution trend is generally reasonably predicted by the VLES and LES models, but the deviation of the prediction and experiment is bigger compared with the mean velocity. Among all the models, the VLES model gives the best prediction, better than the two LES models. Another important observation is that there are relatively large deviations of the prediction with experiment in the near region, i.e. $0.2 \mathrm{~mm}$ and $10 \mathrm{~mm}$, while small deviations at the far downstream regions for all the three models. It implies that, for the turbulence fluctuation (i.e. rms velocities), in the near region, it is highly affected by the inlet turbulence fluctuation, and in the downstream region, it is mainly determined by the swirling flow fluctuation itself. The big difference at the near region may result from the inaccurate inflow turbulence, and the rms velocities are overestimated in the near region generally. For the 


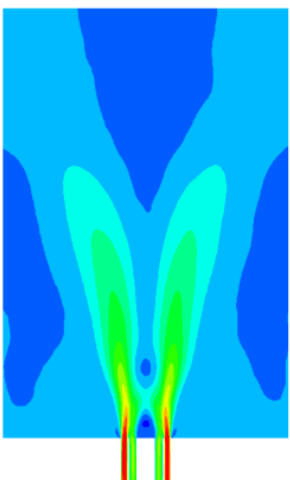

(a)

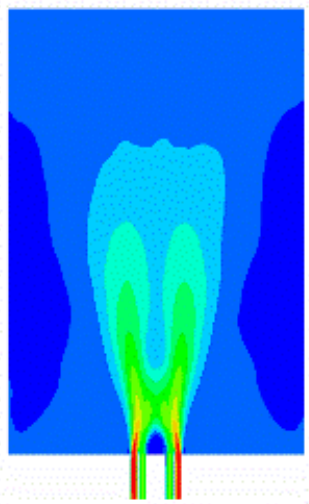

(b)

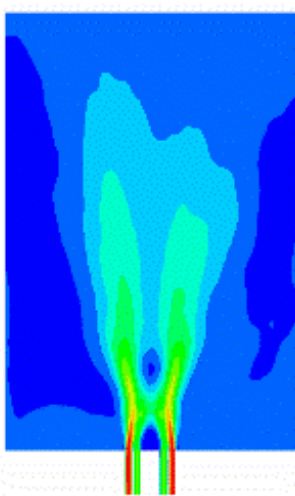

Umean

Umean

Umean

(c)
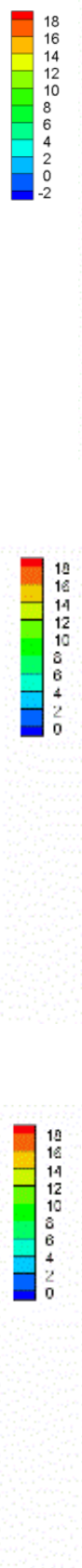

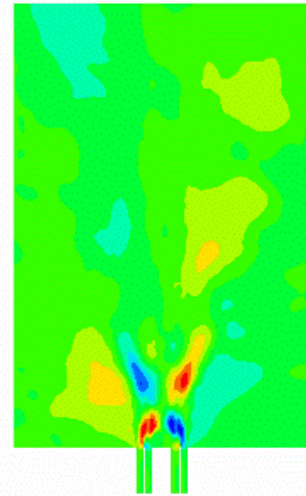

Vmean

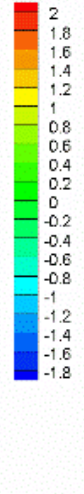

Vmean

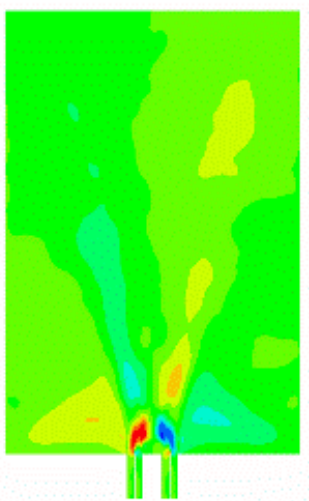

Vmean
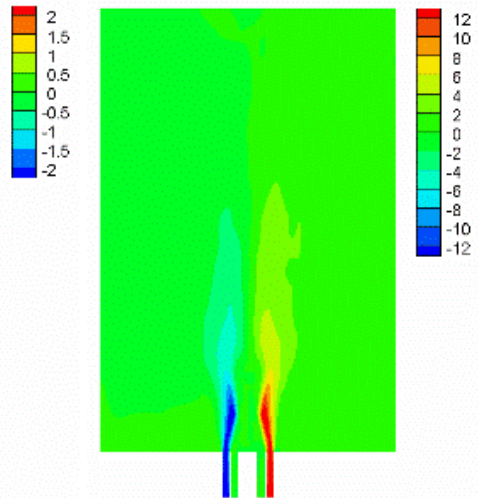

Wmean

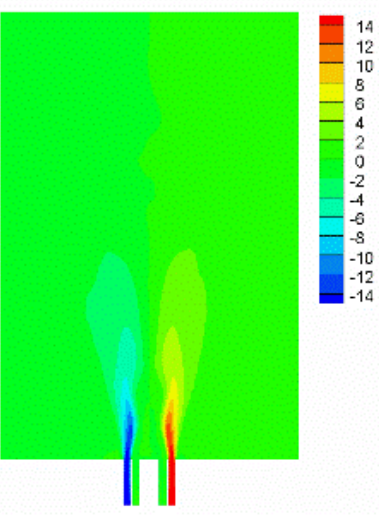

Wmean
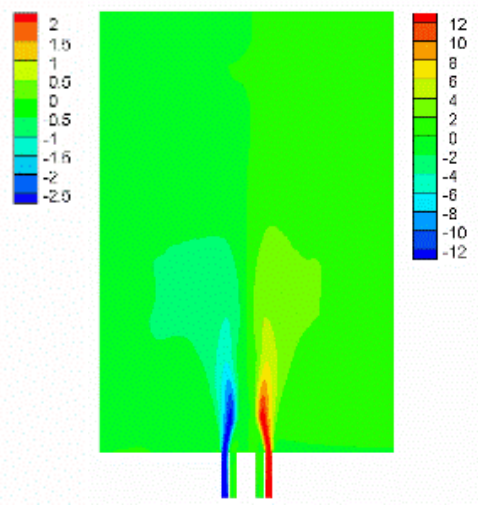

Wmean the swirling turbulent flow with different models: (a) LES-WALE; (b) LES-CSM and (c) VLES.

second location which is $10 \mathrm{~mm}$ away from the inlet, VLES predicts good result along the axial and tangential direction, but, for the radial velocity, the simulation is over-predicted. For the intermediate region at $30 \mathrm{~mm}$ and far region at $50 \mathrm{~mm}$, VLES gives good predictions along the three directions, better than the two LES models. The increase in the vortex structures due to the turbulence generated by the swirling flow makes it more difficult for LES to accurately predict the results at the downstream regions.
Figure 5 shows the contours of the rms velocities by all the turbulence models. Overall, the rms velocity contours agree with each other by different turbulence models, although there are some local differences. It seems that the LES-WALE model (Fig. 5(a)) gives the largest spreading of the swirling jets and the VLES (Fig. 5(c)) and LESCSM (Fig. 5(b)) models produce similar trend of the spreading. For the axial rms velocity at the near region, the results of VLES and LES-WALE are close to each other. For the radial rms velocity, the 

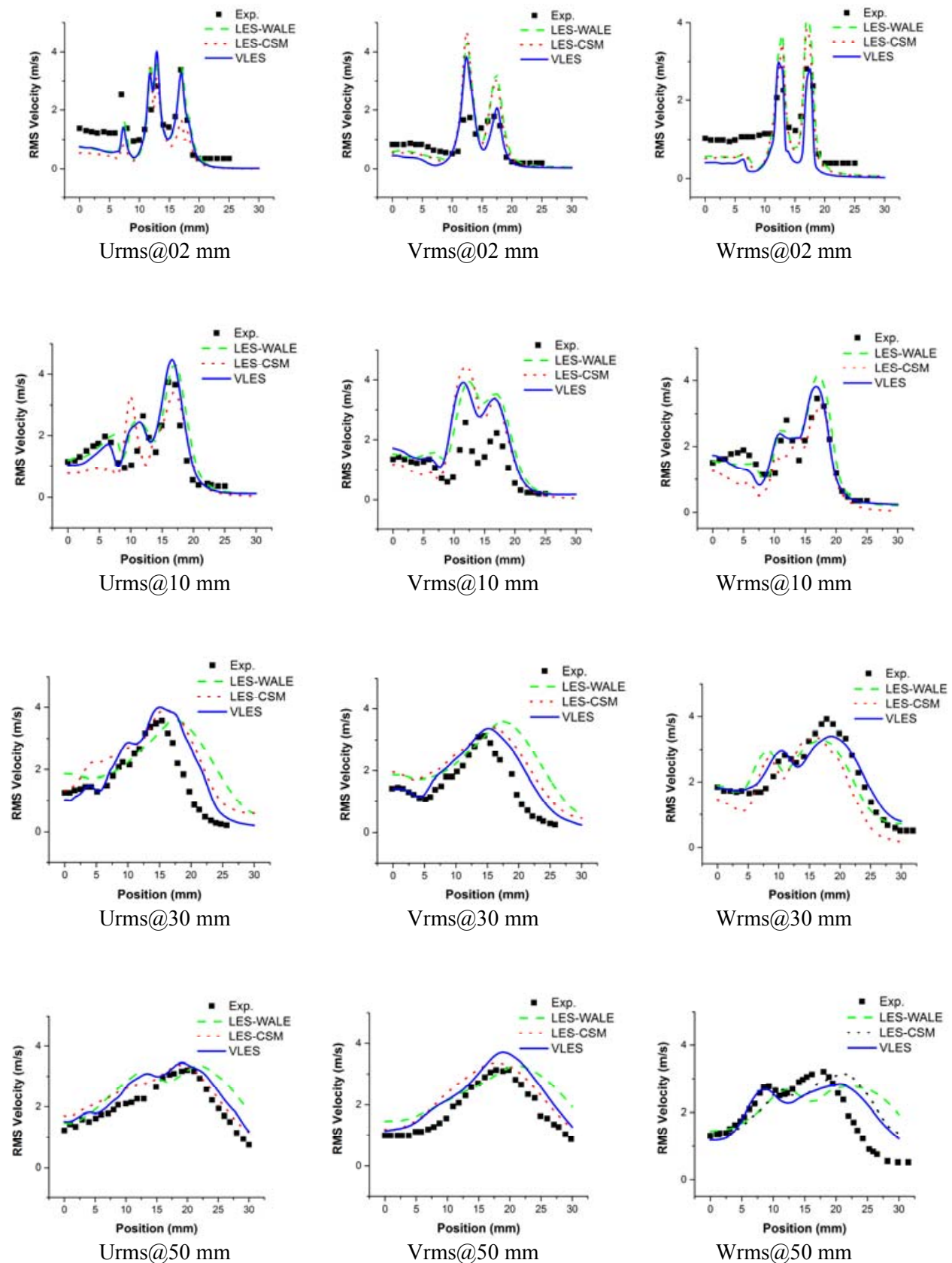

Fig. 4. Comparison of axial (U), radial (V) and tangential (W) rms velocities for the swirling turbulent flow with different models.

VLES gives slightly longer region with large rms velocity value at the near region from the swirling inlet. Combining with the results shown in Fig. 4, the VLES results can be more trusted as the real physical rms flow field of the complex swirling flow, except at the very near region where the rms velocities are over-estimated.

\subsection{Turbulent flow Fields}

Figure 6 shows the Q-criterion results by different models employed to visualize the three-dimensional coherent structures in the swirling flow fields. It can be observed that the flow is dominated by complex turbulent structures with large and small scales, including the vortex breakup, K-H instability, etc. The vortex breakup mainly occurs at the region of the swirling flow development and also the mixing layer regions. The $\mathrm{K}-\mathrm{H}$ instability mainly occurs at the mixing layer regions between different jets. The strong unsteadiness can be clearly observed. There are visual differences between different models. It seems that the VLES results are closer to those from the LES-WALE model, and have slightly large differences from the LES-CSM model. 


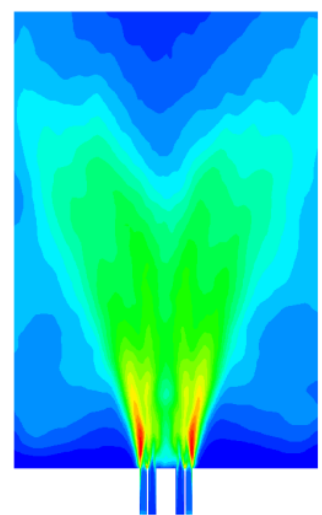

(a)

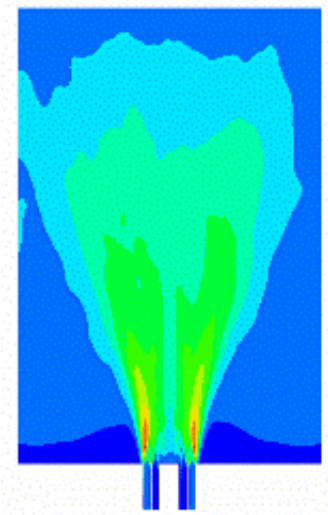

(b)

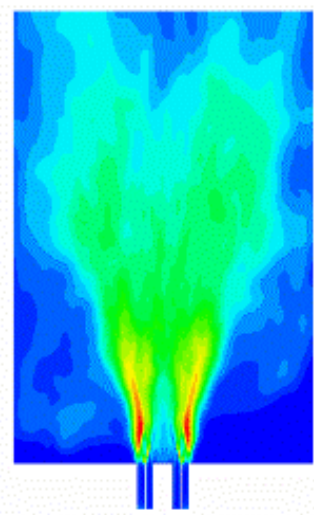

Urms

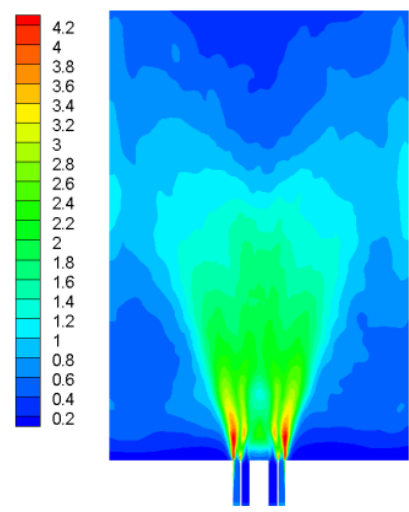

Vrms
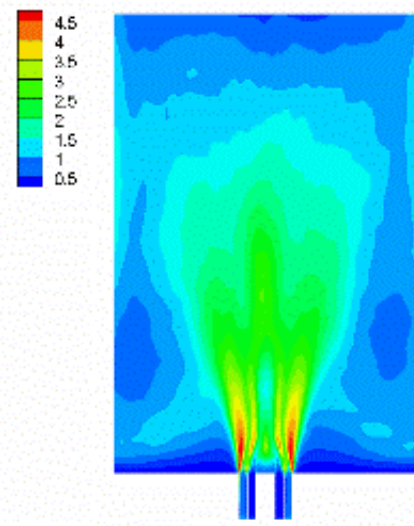

Vrms

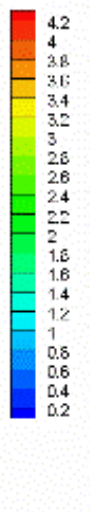

(c)
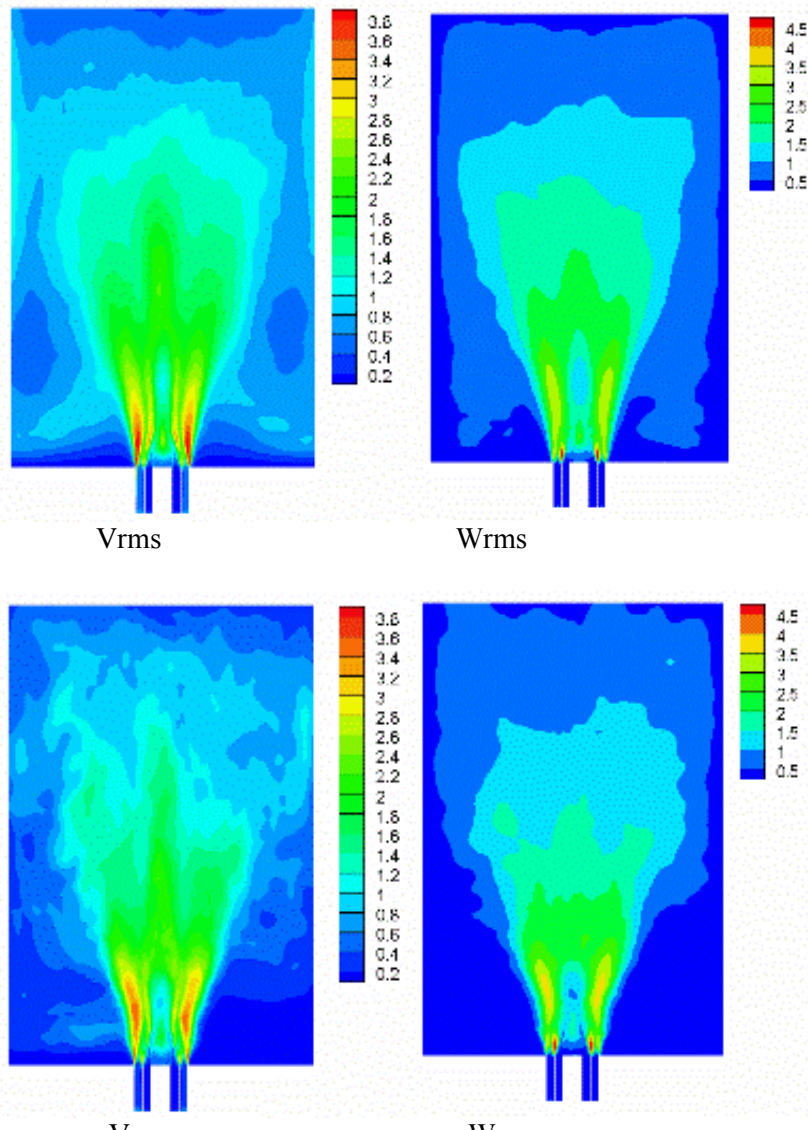

Wrms

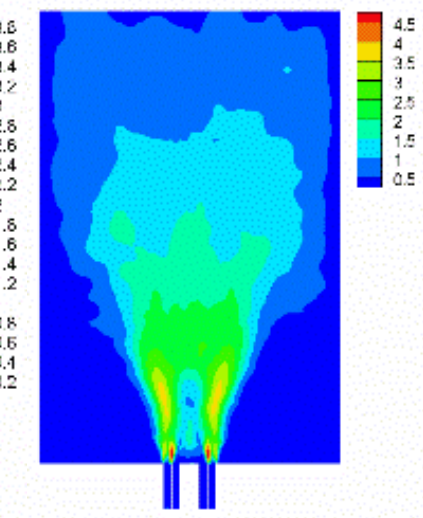

Wrms

ig. 5. Comparison of the contours of the axial (U), radial (V) and tangential (W) rms velocities for the swirling turbulent flow with different models: (a) LES-WALE; (b) LES-CSM and (c) VLES.

Figure 7 shows the instantaneous turbulent viscosity results from different models. All models show small turbulent viscosity values, generally smaller than 10 times of the laminar viscosity (with the value of $1.85 \times 10^{-5} \mathrm{Kg} / \mathrm{m} \cdot \mathrm{s}$ ). It implies that most of the turbulence is directly resolved by the simulation and only small part is modeled by the turbulence model. Another observation is that the distributions of the two LES models (Figs. 7(a) and 7(b)) are similar while they are different from the VLES (Fig. 7 (c)) results. It means that the VLES turbulence modeling is essentially different from the other two LES models.

\section{Conclusions}

A complex turbulent swirling flow after a bluff 

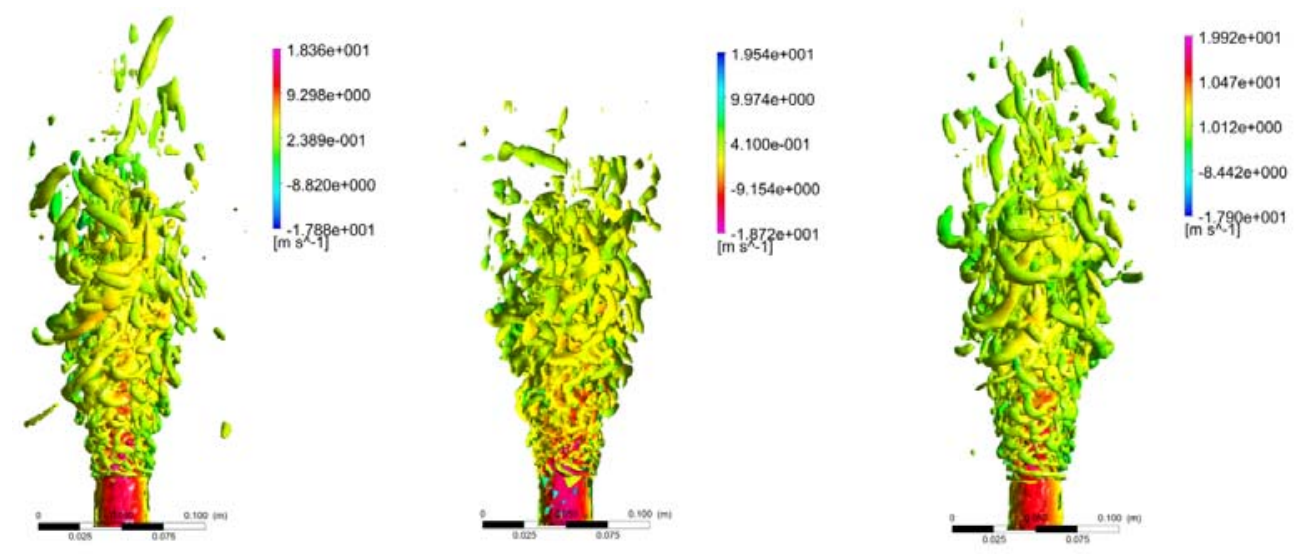

Fig. 6. Iso-surfaces of the second invariant of the velocity gradient $\left(Q=1.0 \times 10^{5} \mathrm{~s}^{-2}\right)$ colored by the instantaneous axial velocity by LES-WALE (left), LES-CSM (middle) and VLES (right) methods.

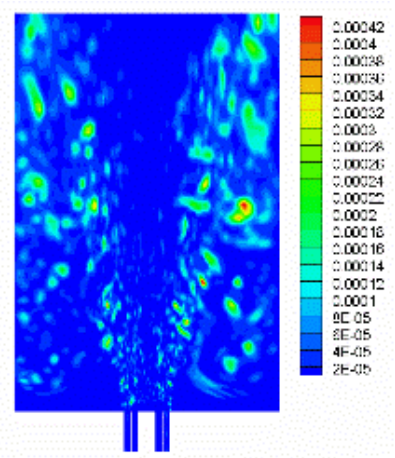

(a)

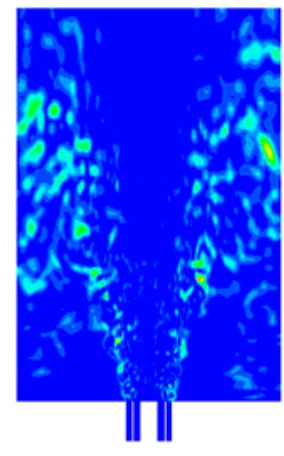

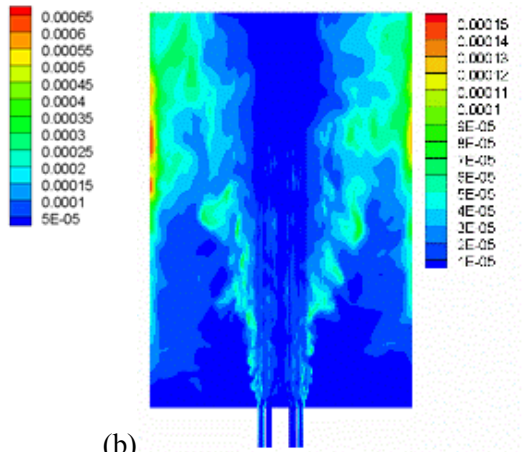

(c)

Fig. 7. Contours of instantaneous turbulent viscosity by LES-WALE (a), LES-CSM (b) and VLES (c) methods.

body is simulated by different turbulent models, including the newly developed VLES model and two classical LES models. The VLES model can work in different turbulence modeling modes covering from RANS to LES and finally approaching DNS mode according to the grid resolution. The capability of VLES for complex swirling flow is validated in details. Mean and rms velocities by the three turbulence models, i.e. LESWALE, LES-CSM and VLES, are compared with the experimental data at various locations. It can be concluded that VLES model has better predictions of the swirling flow field for both the mean and the rms results than the two LES models. For the turbulence fluctuations (i.e. rms velocities), the results at the near region is highly affected by the inlet turbulence, while at the far downstream region, the turbulence fluctuation is mainly dominated by the swirling flow unsteadiness itself. Various fundamental flow mechanisms are well captured by the VLES model, such as the vortex breakup, K-H instability, etc. The study demonstrates that with the help of VLES model, it can lay the foundation for the future high-fidelity computational study of complex swirling turbulent flow in various engineering applications.

\section{ACKNOWLEDGEMENTS}

The work is financially supported by the National Natural Science Foundation of China (grant Nos: 51606095 and 91841302), the National Science and Technology Major Project (2017-III-0005-0029), and the Aeronautics Power Foundation (No. 6141B09050388). X.S. Han acknowledges the support of the Jiangsu Specially-Appointed Professor Program.

\section{REFERENCES}

Al-Abdeli, Y. M. and A. R. Masri (2015). Review of laboratory swirl burners and experiments for model validation. Experimental Thermal and Fluid Science 69, 178-196.

ANSYS Fluent, Theory Guide, Release 16.1, ANSYS, Inc. (2015).

Barlow, R. S., M. J. Dunn, M. S. Sweeney and S. Hochgreb (2012). Effects of preferential transport in turbulent bluff-body stabilised lean premixed CH4/air flames. Combustion and Flame 159, 2563-2575.

Ben-Nasr, O., A. Hadjadj, A. Chaudhuri and M. S. 
P. Tiwari et al. / JAFM, Vol. 13, No. 4, pp. 1107-1116, 2020.

Shadloo (2017). Assessment of subgrid scale modeling for large-eddy simulation of a spatially-evolving compressible turbulent boundary layer. Computers \& Fluids 151, 144 -158 .

Brauner, T., W. P. Jones and A. J. Marquis (2016) LES of the Cambridge Stratified Swirl Burner using a Sub-grid pdf Approach. Flow Turbulence and Combustion 96, 965-985.

Chen, B., K. Ho, Y. Abakr and A. Chan (2016) Fluid dynamics and heat transfer investigations of swirling decaying flow in an annular pipe Part 1: Review, problem description, verification and validation. International Journal of Heat and Mass Transfer 97, 1029 1043.

Girimaji, S. S. (2006). Partially-Averaged NavierStokes Model for Turbulence: A ReynoldsAveraged Navier-Stokes to Direct Numerical Simulation Bridging Method. Journal of Applied Mechanics 73, 413-421.

Gupta, A. K., D. J. Lilley and N. Syred (1984). Swirl flows. Abacus Press, Tunbridge Wells, UK.

Gyllenram, W., H. Nilsson and L. Davidson (2007) On the failure of the quasi-cylindrical approximation and the connection to vortex breakdown in turbulent swirling flow. Physics of Fluids 19, 045108.

Han, X. and S. Krajnovic (2013). An efficient very large eddy simulation model for simulation of turbulent flow. International Journal for Numerical Methods in Fluids 71, 1341-1360.

Han, X. and S. Krajnovic (2015). Very-Large Eddy Simulation Based on k- $\omega$ Model. AIAA Journal 53, 1103-1108

Kobayashi, H. (2005). The subgrid-scale models based on coherent structures for rotating homogeneous turbulence and turbulent channel flow. Physics of Fluids 17, 045104.
Krain, H. (1988). Swirling impeller flow. Journal of Turbomachinery 110(1), 122-128.

Li, J., B. Yu, L. Wang, F. Li and L. Hou (2017). A mixed subgrid-scale model based on ICSM and TADM for LES of surfactant-induced drag-reduction in turbulent channel flow. Applied Thermal Engineering 115, 1322 1329.

Moureau, V., P. Domingo, L. Vervisch and D. Veynante (2010). DNS analysis of a $\mathrm{Re}=$ 40000 swirl burner. Center for Turbulence Research, Proceedings of the Summer Program 2010, 289-298.

Nicoud, F. and F. Ducros (1999). Subgrid-scale stress modelling based on the square of the velocity gradient tensor. Flow Turbulence and Combustion 62, 183 -200.

Saroha, S., S. S. Sinha and S. Lakshmipathy (2018). Evaluation of Partially-Averaged NavierStokes Model method in simulating flow past a sphere. Journal of Applied Fluid Mechanics 11, 1333-1348.

Shah, S. P., S. A. Channiwala, D. B. Kulshreshtha and G. C. Chaudhari (2019). Design, numerical simulation and experimental investigation of radial inflow micro gas turbine. Journal of Applied Fluid Mechanics $12,1905-1917$

Shtern, V. and F. Hussain (1999). Collapse, symmetry breaking, and hysteresis in swirling flows. Annual Review of Fluid Mechanics 31, 537-566.

Sweeney, M. S., S. Hochgreb, M. J. Dunn and R. S. Barlow (2012). The structure of turbulent stratified and premixed methane/air flames II: swirling flows. Combustion and Flame 159, 2912-2929.

Wang, X. T., Z. Ning and M. Lu (2018). Temporal Instability for a Charged Power-Law Liquid Jet in a Coaxial Swirling Air. AIAA Journal $56,3515-3523$ 